\title{
Los retos de la Universidad Peruana en el contexto de la Globalización
}

\section{Challenges of the Peruvian University in the Globalization context}

DOI: $10.46932 /$ sfjdv2n5-030

Received in: Jun 1st, 2021

Accepted in: Sep 30th, 2021

Bazán Linares Magda Verónica

Magister

Institución actual: Universidad Agraria de la Selva

Dirección actual: Jr. Sucre 385: Tingo María

Correo electrónico: magda.bazan@unas.edu.pe

Gaona Portal Milagros del Pilar

Magister

Institución actual: I.E Chuco

Dirección actual: Jr. San Roque 484: Cajamarca

Correo electrónico: milyeduc2019@gmail.com

\section{Luis Grados Carlos Alberto}

Magister

Institución actual: Universidad Católica de Trujillo

Dirección actual: Av. Moche 843, Urb. Santa María: Trujillo

Correo electrónico: cluis@uct.edu.pe

\section{Luna Acuña Maleyne Lisseth \\ Magister}

Institución actual: I.E. 83004 - Ex 91

Dirección actual: Jr. Amalia Puga 338 - Cajamarca

Correo electrónico: 1maleynelisseth@gmail.com

\section{Peralta Roncal Liliana Ethel}

Magister

Institución actual: E. № 16081 "Señor de Huamantanga" - Jaén

Dirección actual: Pasaje Uno $\mathrm{N}^{\circ} 227$-Urbanización El Edén - Jaén

Correo electrónico: lilianaperaltaroncal@gmail.com

\section{José Ángel Meneses Jiménez}

Doctor

Institución actual: Universidad Nacional de Ucayali

Dirección completa: Car. Federico Basadre KM. REF: Ucayali Coronel Portillo-Calleria

Correo electrónico: jose_meneses@unu.edu.pe

\section{RESUMEN}

La globalización es el fenómeno económico que impacta en diversas esferas de una sociedad, en las cuales genera numerosos cambios, siendo una de ellas el campo educativo. La educación, especialmente la 
universitaria ha tenido que adaptarse a estos cambios con el propósito de continuar brindando un servicio de acorde a las necesidades del mundo globalizado manteniendo su visión humanista, científica y tecnológica.

Las universidades peruanas sumándose a estos cambios asumieron el reto de la virtualización de sus sistemas de enseñanza aprendizaje, adaptando modelos pedagógicos, estrategias metodológicas y uso de recursos tecnológicos a las nuevas necesidades de una educación no presencial, en donde los docentes y estudiantes fortalecieron sus competencias digitales haciendo uso de diversos recursos tecnológicos disponibles en la web.

La UNAS al igual que las diferentes universidades nacionales e internacionales asumió el reto de una educación no presencial durante la emergencia sanitaria, este paso significó un cambio de su modelo pedagógico, adaptando la currícula académica al desarrollo de competencias digitales, al uso de estrategias, recursos y herramientas disponibles on line; todo ello a partir del análisis de sus procesos institucionales, administrativos, académicos y de investigación. Convirtiéndose así en ese ente dinamizador y de cambio que exige la globalización.

Palabra clave: Universidad, Globalización, Tecnología.

\begin{abstract}
Globalization is economic phenomenon that impacts diversity spheres of a society, in which it generates numerous changes, one of them being educational field. Education, especially university education, has been adapted to these changes in order to continue providing a service in accordance with needs globalized world, maintaining humanistic, scientific and technological vision.

Peruvian universities, joining these changes, took on the challenge of virtualizing their teaching learning systems, adapting pedagogical models, methodological strategies and use of technological resources to new needs of virtual education, where teachers and students strengthened their skills making use various technological resources available on web.

UNAS like different national and international universities, has assumed the challenge of virtual education during health emergency, this step has been meant a change in its pedagogical model, adapting academic curriculum to development of digital skills, the use of strategies, resources and tools available online; all this from the analysis of its institutional, administrative, academic and research processes. Thus, becoming that dynamic and changing entity that globalization requires.
\end{abstract}

Keywords: University, Globalization, Technology.

\title{
1 INTRODUCCIÓN
}

La globalización es un fenómeno esencialmente de carácter económico que sustenta su accionar en la interconectividad de los países, en donde se eliminan y/o reducen las barreras arancelarias para dar paso al intercambio de bienes y servicios entre diferentes naciones; pasando de una economía local a una mundial. Este fenómeno económico tiene un impacto en las diferentes esferas de una sociedad, en lo político con la promoción de una democracia liberal, en lo social con la reivindicación de los Derechos Humanos, en lo tecnológico con el avance del Internet y en lo educativo con el desarrollo de un modelo neoliberal que promueve el logro de competencias para la empleabilidad, el uso de la virtualidad y las TIC para un mundo global. 
Uno de los aspectos en donde la globalización ha tenido mayor impacto en la última década del s. XXI es la educación, pues ha permitido cuestionar los procesos tradicionales de enseñanza aprendizaje para un mundo global, ofreciendo nuevos modelos pedagógicos que promocionan y priorizan habilidades diversas tales como el trabajo en equipo, la empatía, la resiliencia, la alfabetización digital por encima de la transmisión de conocimientos, haciendo uso de diversas herramientas digitales, en donde la tecnología, el internet y la virtualidad se han convertido en aliados para nuevos procesos de producción de conocimientos a escala mundial, en donde las redes sociales, las comunidades de inter aprendizaje virtual están ofreciendo una nueva visión de la educación, promoviendo la transformación de roles de los individuos que están inmersos en los procesos educativos.

Esta última aseveración lo hemos vivido con la pandemia de la COVID-19, en donde los sistemas educativos del mundo han implementado modelos pedagógicos que tengan como base la virtualidad, el acceso remoto, la no presencialidad de la educación, donde los actores educativos tal es el caso de los estudiantes y docentes han tenido que potenciar sus habilidades digitales a esto se suma la actitud de los docentes y estudiantes, que evidencian la importancia y necesidad de aprender el uso de recursos tecnológicos para estar acorde a las necesidades educativas que ofrece un mundo global.

La UNAS, situada en la cuidad de Tingo María, distrito de Rupa- Rupa, brinda un servicio educativo a 3010 estudiantes en 6 carreras profesionales de alta demanda laboral. Siendo esta casa universitaria la pionera en la concreción de compromisos morales y ambientales, aportando a la formación de estudiantes proactivos e innovadores que contribuyan con el desarrollo del país. Frente a los cambios del mundo globalizado que ha afectado a todos los sectores, la Universidad no ha estado exenta de estos procesos y ha tenido que adaptarse a este contexto. La UNAS siguiendo los lineamientos y políticas que supervisan el funcionamiento de estas casas de estudio ha adaptado su estructura curricular para estar acorde a las exigencias y requerimientos profesionales que se necesitan para hacer frente a la empleabilidad del mundo globalizado.

Concluimos resaltando la importancia del análisis PESTEL en nuestro informe académico, el cual permitió identificar las necesidades educativas de la UNAS en el proceso de globalización en los aspectos político, económico, social, tecnológico y ecológico, planteando frente a ellas, el desarrollo de alianzas estratégicas con Instituciones locales e internacionales, que en trabajo conjunto favorecerán la calidad educativa de los estudiantes, la preparación y actualización docente para el trabajo virtual, semipresencial y presencial en el tiempo de pandemia y post pandemia. 


\section{OBJETIVOS}

La presente investigación tuvo como objetivo general:

Implementar acciones pedagógicas y de gestión para la atención de los estudiantes y maestros en la educación virtual, semipresencial y presencial en el contexto de la pandemia, manteniendo la calidad educativa de la UNAS.

Siendo los objetivos específicos los que se detallan a continuación:

- Promover alianzas estratégicas con entidades nacionales e internacionales para promover el intercambio de aprendizajes y experiencias educativas en los estudiantes.

- Implementar e institucionalizar en los documentos de gestión un currículo centrado en los aprendizajes y competencias digitales para docentes y estudiantes.

- Diseñar y ejecutar un plan de acción para el trabajo virtual, semipresencial y presencial en el tiempo de pandemia, considerando las normas de bioseguridad e involucrando a todos los agentes educativos.

- Promover la práctica de conductas ambientales responsables en la comunidad unasina y tingaleza mediante la ejecución del plan ambiental.

\section{MARCO TEORICO}

\subsection{LA GLOBALIZACIÓN}

La globalización es un acontecimiento relativamente reciente que ha calado rápidamente en todos los aspectos de las actividades del ser humano, esta globalización se ha dado fundamentalmente en materia económica. Según Hernández (2020) refiere que cuando el mundo viaja a una era posmoderna, se abre paso a un nuevo modelo económico, científico, y también nuevas tecnologías y esto abren camino a cambios vertiginosos en los aspectos también culturales.

Este fenómeno llamado globalización se considera como un concepto parecido a la de "integración económica global" (Lombaerde \& Lapade, 2012). Este fenómeno ha surgido para permanecer y acompañarnos por un tiempo prolongado, afectando la vida de los habitantes.

La globalización desde un punto de vista económico tiene influencia en los procesos productivos, distribución de bienes, servicios, desde el aspecto político tiene que ver con el nacimiento de una sociedad diferente en la cual las interrelaciones políticas, sociales y económicas se transformen. En el aspecto social está evolucionando según las nuevas tecnologías de la información evolucionan propiciando la interculturización al mantener interconectados con el resto del mundo. Se puede concluir que el mundo cambió y está cambiando constantemente y que depende de la capacidad del hombre para adaptarse a estos nuevos contextos y se convierte en el actor fundamental de este proceso. 
Desde el campo de la educación es la que dará el soporte para estar abiertos a estos cambios sociales, políticos y sobre todo a la virtualización de la educación para estar en sincronía con los cambios y retos del mundo globalizado.

\subsection{LA EDUCACIÓN CON RESPECTO A LA GLOBALIZACIÓN}

La eficacia en los modelos de educación está incrementándose a nivel internacional y el currículo se vuelve más complejo y la educación especialmente la superior, debe capacitar, dotar y entrenar a los estudiantes en el manejo de tecnologías novedosas, así como también formarlos en varios idiomas.

Estamos viviendo una revolución en el conocimiento en la cual hay un crecimiento muy significativo en el incremento del conocimiento del hombre. En este proceso se enfatiza la jerarquía de la elaboración del conocimiento funcional Gibbons et al. (1998) "la creación del conocimiento posee el objetivo de ser útil a alguien, pudiendo ser en lo industrial, a nivel de gobierno, socialmente; el conocimiento se ejecuta desde el aspecto de negociación constante se produce en la media que se involucren e incluyan los intereses de los actores involucrados".

Estamos continuamente siendo testigos de los constantes adelantos tecnológicos y científicos. Esto surge como una consecuencia del fenómeno de la globalización tanto en el aspecto económico, como cultural. También es notoria la influencia de la información mediante los medios virtuales, este tipo de información se ha visto masificada en todos los estratos sociales lo que ha contribuido a mejorar los canales de comunicación.

En la actualidad las naciones miden como uno de los parámetros de riqueza la generación de conocimientos nuevos y novedosos en el campo de la informática, bioingeniería, la genética, nanotecnología entre otros. Las políticas gubernamentales de países en vías de desarrollo están sometidos a la presión de incrementar el gasto en temas referidos a la educación, esto con miras a contar con individuos cada vez más calificados y capacitados para desempeñarse y ser capaces de producir en el contexto que la globalización exige.

Hoy en día se ha visto una gran competitividad por la mejora en la calidad educativa nivel mundial, en la cual hay la exigencia que la educación superior debe formar a los estudiantes en las nuevas estrategias para la promoción de las competencias digitales.

La virtualización de la educación ha significado que ésta se desarrolle rápidamente y debe expandirse conllevando con ello a bajar los costos mediante la implementación de un tipo de educación virtual y que tiende a convertirse en una modelo predominante en el ámbito universitario. Estos son las implicancias del modelo globalizado en la cual debe darse necesariamente un cambio en el aspecto cultural en la cual los excluidos puedan acceder a una buena formación académica. 
Según Latinobarómetro (2018), en Latino América hay un 60 \% de la población en 17 países que piensan que lo que determina en éxito en la vida es el nivel de educación. También en dicho informe se decía que para el 2020 los conocimientos se doblarán cada 73 días en educación, provocando un cambio importante en el mercado laboral. El BID afirma que en los últimos años hay un incremento sustancial de estudiantes matriculados en la universidad.

Aún cuando las características sociales actuales la educación peruana hasta antes de la pandemia ha estado muy estancada en viejos paradigmas y muy ajena a las nuevas tecnologías. En este contexto los docentes universitarios no son ajenos a estas realidades pues a decir de algunos autores se seguían utilizando un modelo desarrollado en el siglo XIX. Una educación masificada es por ello por lo que la educación universitaria necesita asumir nuevos retos, hacer uso de las nuevas tecnologías, brindar una educación que responda a las exigencias que señala y exige los nuevos retos de la tecnología y el mundo globalizado.

Debemos abandonar la práctica de la de la educación en la que el estudiante recibe información de manera pasiva, regidos por programas ya establecidos donde se tiende a la memorización de los conocimientos. Se debe apostar por una educación que sea capaz de producir conocimiento, ellos implican mejorar los sistemas de gestión, incrementar la inversión financiera, mejorar sustancialmente los sistemas de evaluación y el currículo. Se debe apostar por una transformación para contribuir a generar un clima de colaboración y cooperación en el que predomine la igualdad y no dejando de lado la modernidad.

El acceso a la educación es un derecho fundamental y un derecho a una educación de calidad, para poder enfrentar los retos que trae el contexto del siglo XXI, con la constante actualización de los contenidos a las nuevas tecnologías informáticas.

La UNAS en su plan estratégico institucional 2019 - 2023 dentro de sus objetivos estratégicos institucionales contempla el mejorar la calidad de formación académica de los estudiantes apostando a un silabo por competencias actualizados, currículos actualizados e implementados en un $92 \%$, acreditar a las escuelas académicas existentes de un $0 \%$ del 2017 a un 50\% en el 2023\%, mejora se servicios, equipamiento e infraestructura pasar de un 75\% del 2017 a un 100\% al 2023, fortalecer la investigación formativa científica tecnológica, innovadora y humanística, promover centros de investigación implementados para la comunidad educativa. Fortalecer el liderazgo, posicionamiento, conectividad con los grupos de interés.

Durante la pandemia en el 2020 la universidad implementó la plataforma virtual Ms Teams, Cisco para el desarrollo de las actividades académicas, ha tenido que difundir masivamente el uso de la biblioteca y el aula virtual que solo algunos docentes y estudiantes lo utilizaban, pero no se utilizaba de forma masiva. La utilización de esta plataforma permite desarrollar las clases de manera sincrónica y en 
tiempo real con los estudiantes y ha permitido el intercambio de información y capacitaciones constantes de los docentes en el manejo de estas herramientas virtuales.

\subsection{LA UNIVERSIDAD AGRARIA DE LA SELVA}

En la cuidad de Tingo María, Rupa-Rupa se sitúa la UNAS, provincia Leoncio Prado, correspondiente a la región Huánuco. Su ubicación geográfica es de latitud sur 9¹7'08" y longitud Oeste 75 59'52", a $660 \mathrm{msnm}$, cuya temperatura promedio es de $24^{\circ} \mathrm{C}$. La población es de 46191 habitantes hasta el año 2017, dentro de las principales actividades económicas está el comercio, turismo y agricultura destaca el cultivo del café, cacao y coca. En las últimas décadas se ha incremento la demanda en el sector turismo, siendo un lugar preferido de los turistas nacionales y extranjeros.

La Universidad alberga un total 247 docentes y 3010 estudiantes en seis facultades: Ciencias Económicas y Administrativas, Zootecnia, Agronomía, Ingeniería en Industrias Alimentarias, Recursos Naturales Renovables y sus respectivas escuelas, ingeniería Informática y Sistemas. Las carreras profesionales con mayor demanda son las de Ingeniería Mecánica Eléctrica, Ingeniería Informática y Contabilidad.

La UNAS al 2021 se propone ser una institución que lidera la formación de futuros profesionales, éticos y niveles altos de competitividad, con elevado compromiso en gestión ambiental y exhaustivo para el progreso sustentados que contribuyan con el progreso de la nación.

El campus universitario se ubica en la carretera central Km. 1.21, una extensión aproximada de 301 ha; en la cual se encuentran diversas instalaciones para llevar a cabo las actividades académicas y de carácter científico. Cuenta con el Centro de Investigación (CIUNAS), espacios de lectura, amplios laboratorios, área de comedor y hospedaje para estudiantes.

La UNAS cuenta con módulos de actualización, experimentación y producción orientada a la instrucción de los estudiantes, exploración de diferentes variedades agrícolas, boscosos, prados, así también como granja para la crianza de animales menores, ganado vacuno como parte de la investigación pecuaria. Estas acciones académicas giran en torno a tres ejes fundamentales: Primero que faciliten actividades de índole académico, indagación y proyección a la comunidad, segundo son los ambientes para la experimentación e indagación científica, tercero, la conservación y producción de especímenes forestales.

Dentro de las actividades culturales cuenta con un Jardín Botánico con plantaciones de Bambú Asiático y Cacao, centro de idiomas y Pre-universitario, centro de datos de información forestal, la oficina de investigación y en los últimos años se ha invertido en adquisición de equipos de última generación para 
implementar los laboratorios. En lo referente a esparcimiento y recreación cuenta con un complejo destinado a la práctica de diferentes disciplinas deportivas.

En relación a los ocho objetivos del milenio, la UNAS forma profesionales que aportan y contribuyen con el crecimiento regional y del país, tiene convenios interinstitucionales con universidades del país y universidades extranjeras, para realizar pasantías y estudios investigación y pos- grado.

A largo plazo se configurará el Plan Bicentenario, que reúne las propuestas en materia de política, con el objetivo de alcanzar el desarrollo sostenible del país en un lapso de 10 años. Por lo que la UNAS ha acogido dicho plan, orientándose en los seis ejes estratégicos relacionados a las oportunidades, accesos a los servicios y los derechos de la persona; Estado y gobernabilidad; economía, competitividad y empleo; así como el progreso regional, aspectos relacionados a la infraestructura y RRNN y medio ambiente. En este marco del Plan Bicentenario la UNAS promueve el desarrollo tecnológico a través de sus planes curriculares, implementando, promoviendo el uso plataformas virtuales y capacitando al personal docente, administrativo y estudiantil en el manejo de las herramientas tecnológicas.

Antes de la pandemia, la Universidad desarrollaba sus actividades académicas de manera presencial en los diferentes ambientes y horarios del campus universitario atendiendo de manera regular a la población estudiantil y cumpliendo los objetivos institucionales. A partir del mes de marzo del 2020, la UNAS dando cumplimiento al plan de emergencia sanitaria con la intención proteger la salud de docentes y estudiantes mediante acuerdo del consejo universitario decide suspender las clases presenciales e iniciar el proceso de adecuación y adaptación a la enseñanza virtual.

En repuesta al DL $\mathrm{N}^{\circ}$ 1496, con fecha 2020-05-10, publicado en el Diario Oficial El Peruano, la Universidad inició el proceso de implementación para la atención virtual de los estudiantes; para ello se habilitó las plataformas Ms Teams, Cisco, Cisco webex, Aula virtual, Biblioteca virtual y dotando a los docentes de los recursos y herramientas digitales necesarias para el desarrollo de las clases virtuales.

A partir del segundo semestre de 2020, se firmaron alianzas estratégicas para fortalecer las competencias digitales de los docentes y estudiantes uno de estos fue el Plan de apoyo en la implementación y diseño de acciones metodológicas para atender a los estudiantes de educación superior universitaria públicas del Perú realizado entre el MINEDU y el BID.

El 2021 se inició con el proceso de admisión para el año lectivo 2020-II siendo este de forma presencial poniendo en práctica las medidas de bioseguridad con la colaboración del comité COVID integrado por los docentes y el centro médico de la universidad.

La plana docente que se capacitó en el plan de fortalecimiento, actualmente desarrolla las clases sincrónicas y asincrónicas mediante diferentes plataformas y medios virtuales y tecnológicos brindados por la universidad; sin embargo, tan solo el 15\% de los docentes cumplen con la virtualización de sus 
asignaturas. Para el último trimestre del 2021 la Universidad pretende que el 50\% del total de docentes hayan dado cumplimiento con la virtualización de sus cursos.

Para el 2022, dado que en el proceso de vacunación se está incluyendo a los jóvenes, se prevé el retorno progresivo de las actividades académicas en la modalidad semipresencial de las asignaturas que ameriten el desarrollo de prácticas de laboratorio; esto está supeditado al estado de emergencia que se puedan presentar por los contagios de las nuevas variantes de la COVID como son la variante Delta, la variante $\mathrm{Mu}$ y otras variantes que se puedan desarrollar.

El presente proyecto denominado "Los retos de la Universidad Peruana en el contexto de la Globalización”, surge como una observación durante la atención remota a los estudiantes, donde se detecta que los docentes no han realizado los procesos de adecuación a la virtualidad de manera óptima, debido al desinterés, escaso dominio de la metodología y estrategias pedagógicas para una enseñanza virtual, escasa disposición hacia el uso de las herramientas digitales y plataforma Ms Teams por parte de los docentes y limitado interés hacia el desarrollo de las competencias digitales.

Para analizar la problemática hemos creído conveniente hacer un análisis del entorno general de la UNAS haciendo uso de la técnica PESTEL, con el cual hemos evidenciado el siguiente diagnóstico.

En el aspecto P-Político. Según el Diario Oficial El Peruano (2020) en el Decreto Supremo Nº022021 MINEDU que orienta las características aquellos que serán acreedores al beneficio de servicio de internet en diferentes universidades públicas, según el Presupuesto del Sector Publico para el Año Fiscal 2021, Ley 31084, Articulo $86^{\circ}$.

En lo E-Económico. Como ya es de conocimiento mundial, la pandemia ha venido provocando crisis económica a nivel mundial. El Perú no es ajeno a esta crisis, según el INEI en el año 2020, la economía en el Estado Peruano ha tenido un declive del producto bruto interno (PBI) del 29.8\% en el segundo trimestre del año antes mencionado, en cambio el tercer trimestre sólo del 9.4\%; asimismo de tener un acrecentamiento en lo referente a la tasa de desempleo del $40.2 \%$ conllevando a una reducción significante de los ingresos de la población económicamente activa.

La crisis económica ha situado a varios centros de estudio universitarios en situación de desventaja; las universidades privadas, las que más han sufrido esta crisis son las que dependen de los ingresos económicos por matrícula y pensiones de parte de los estudiantes, tal como lo señala la asociación de instituciones privadas de educación superior; diez de estas universidades privadas estarían por suspender sus actividades académicas por el déficit en las matrículas, la deserción y la morosidad han puesto en riesgo a estas universidades. Respecto a las universidades públicas, que por seguir el proceso de licenciamiento (SUNEDU) antes de la pandemia, invirtieron en infraestructura para construcción de 
laboratorios, contratación de personal docente y administrativo, como también de bienes y servicios conllevándolas a situación de vulnerabilidad.

En el aspecto S-Sociale. El abrupto corte de las labores académicas universitarias ocasionadas por la COVID-19, ha dejado en evidencia le deficiente sistema educativo en materia de tecnologías y en los países tercermundistas. Este contexto, incorporado los bajos niveles en educación en secundaria, evidenciado por medio por los resultados obtenidos en la prueba realizado en 2018 MINEDU (2019), estamos ubicados en el 64 lugar de 77 naciones, reflejando muchas carencias que se tiene que solucionar.

Si se desea potenciar los niveles de la formación universitaria, es preciso trabajar y optimizar la calidad en la educación básica, para certificar el desarrollo de capacidades estudiantes así lo enfatiza Miguel Urquiola en el Webinar (16/07/2020) del BID: “COVID-19 y Educación: El efecto disruptivo de la pandemia en la educación superior"

Relacionado con la formación universitaria, las consecuencias disruptivas de la COVID-19, se aprecian en que la enseñanza aprendizaje virtual, muestra ineficacia ya que estas instituciones no estaban implementadas con la herramienta tecnológicas necesarias para la atención de los estudiantes en el trabajo remoto. Hay una diferencia en la capacidad y logística de los docentes para adaptarse a los entornos virtuales. Se hizo necesario virtualizar los contenidos impartidos en forma presencial, generando un ambiente de desequilibrio profesional en los docentes al no estar preparados para afrontar los nuevos retos tecnológicos.

En lo T- Tecnológico. Según Álvarez (2020) en el estado de cuarentena por Covid 19, las empresas necesitaron atender con mayor fuerza a la población y así sostenerse en el mercado con la venta de herramientas digitales, ayudando con las necesidades tecnológicas de las personas. En esta pandemia el trabajo se volvió virtual, siendo necesario tener acceso a la tecnología para el trabajo a distancia, evitando así el contacto físico que implica riesgos.

Estudios realizados por la compañía EY en el 2019 referente a la innovación en materia de tecnología en el país, reveló que el $27 \%$ las entidades poseen un adelanto incipiente, un $63 \%$ están orientadas en un proceso de adelanto tecnológico, y el 10\% logró estándares relevantes desarrollo digital. Siendo los rubros que mayor desarrollo evidenciaron son los que tienen que ver con las empresas dedicadas a las comunicaciones con un $68.3 \%$, las agencias bancarias y las aseguradoras el 63.2\%, y las dedicadas a brindar Servicios 62.7\%. Por lo tanto, se evidencias las preponderancias de estas tres organizaciones se encuentran altamente enforcadas y en el contexto actual, están formulado procesos de adecuación importante empleando conocimientos e instrumentos digitales para afrontar la actual emergencia sanitaria. 
El sector educación enfrentó un enorme reto al involucrarse en el proceso de la virtualización de la educación, todo en el cumplimiento de las medidas sanitarias en el marco de la pandemia.

- Una brecha digital y escaso dominio de las tecnologías.

- Limitados conocimientos en cuanto a instrumentos de evaluación que evidencien los aprendizajes en el contexto de la virtualización de la educación.

- Pocos docentes aptos para brindar educación virtual y la importancia de la acreditación.

En el aspecto E- Ecológico. Según CEPAL (2020), la COVID 19, ha promovido las medidas de cuarentena, las cuales provocaron una reducción en el uso de vehículos motorizados, lo que causó una disminución en el uso de combustibles, logrando que sean menos las emisiones de gases y la contaminación ambiental. Los recursos naturales son indispensables en esta pandemia como los alimentos, siendo la actividad agrícola muy importante. El agua potable, es fundamental para las medidas de lavado de manos, que ayudan a evitar los contagios; cabe resaltar que en la lucha contra la pandemia contar con agua potable es muy necesario; sin embargo, el 26\% de los habitantes del Caribe y Latino América (166 millones de seres humanos) no cuentan con agua potable para el consumo humano, siendo el 58\% para la población rural que no cuenta con este factor abiótico; también la energía y la electricidad garantizan el funcionamiento de hospitales y las actividades en el hogar.

La Agenda 2030 y los fines de biodiversidad, promueven que se proteja la biodiversidad para garantizar los servicios de la población de manera sostenible; así como garantizar la alimentación a la población en situación de vulnerabilidad.

En lo L- Legal. El D.L. $\mathrm{N}^{\circ}$ 1496, publicado en el Diario Oficial El Peruano 2020, instaura las Disposiciones en Materia de Educación Superior Universitaria en el Marco del Estado de Emergencia Sanitaria Nacional. Art. $3^{\circ}$ del D.L. N. 1496 , SUNEDU autoriza el ejercicio de programas, de las universidades licenciadas, en la forma semipresencial y a distancia, cumpliendo lo establecido en el marco de la emergencia sanitaria.

La Resolución N²06-2020-CU-R-UNAS fija el inicio de las clases virtuales del semestre académico 2020-I.

\subsection{PROPUESTA DE ESTRATEGIAS PARA MEJORAR LA EDUCACIÓN CON RELACIÓN A LOS EFECTOS DE LA GLOBALIZACIÓN}

A continuación, después de análisis PESTEL, se proponen las siguientes estrategias frente a las necesidades encontradas, para la mejora de la educación en la UNAS con relación a los efectos de la Globalización. 
En lo P-Político se identificaron las necesidades siguientes, escasa implementación de políticas de Estado a largo plazo para la promoción de los recursos tecnológicos en las universidades. Limitado interés en la implementación de políticas de gobierno para el desarrollo y promoción ciencia y tecnología.

Ante esta necesidad se propone como estrategias la implementación de políticas universitarias a través del vicerrectorado de investigación y el vicerrectorado académico para promocionar y desarrollar la tecnología y la ciencia.

Promover alianzas estratégicas con entidades universitarias del país y con otras de índole internacional que permita pasantías de estudiantes, becas, entre otras.

En lo E-Económico se encontró como necesidad que la universidad ha sufrido un impacto económico negativo en sus ingresos económicos al ver limitado el uso de los servicios que brindan a terceros producto de la paralización de las clases y el trabajo presencial en emergencia sanitaria.

La estrategia sugerida es la adecuación de los servicios presenciales que ofrece la universidad a un entorno virtual en forma permanente a través de diferentes plataformas virtuales y modalidades de aprendizaje, e institucionalizarlo en los documentos de gestión de UNAS.

En el plano S-Sociales se reconoció la necesidad ocasionada por el cierre de las clases presenciales que los estudiantes en situación de vulnerabilidad socioeconómica se vieron afectados al no contar con los servicios de comedor universitario, albergue, servicio médico, psicológico.

Los docentes y el personal administrativo presentaban cuadros de ansiedad y preocupación al no saber cómo lidiar con situación incierta y retadora que demandaba esta coyuntura sanitaria.

Ante ello consideramos que es importante la planificación y ejecución de programas de soporte emocional virtual, con el propósito de salvaguardar el equilibrio emocional de la comunidad universitaria y ejecutar de forma permanente las actividades programadas en el protocolo de bioseguridad frente a la COVID-19.

En el entorno T-Tecnológico pudimos notar un Limitado acceso y conocimiento del uso de las nuevas TICS y el escaso acceso a los recursos tecnológicos fue una dificultad para el inicio y desarrollo trabajo virtual en el contexto de la crisis pandémica.

Para contrarrestar estas falencias se debe Implementar de un diseño curricular centrado en el aprendizaje y basado en las competencias digitales para el uso en tecnologías de información, impulsando un trabajo colaborativo. Incluir en el documento de gestión como parte de los objetivos estratégicos un plan para el logro de competencias relacionadas con el manejo de la tecnología en los docentes.

En el ámbito E-Ecológico se observó la escasa participación y promoción del cuidado, ahorro del agua, manejo de residuos sólidos, adecuado uso de energía por parte de la comunidad universitaria mediante las plataformas virtuales. 
Ante esta realidad se propone monitorear la ejecución del plan ambiental que genere la práctica de conductas ambientales responsables en la comunidad Unasina y la comunidad tingaleza. Realizar campañas de propagación, concientización y capacitación en temas concernientes al manejo de desechos sólidos, a través de los diferentes medios y/o canales de difusión.

En el campo L-Legal se visualizó la carencia de un plan institucional a largo plazo que aborde el tema del trabajo remoto por la mergencia sanitaria según el D.L. $\mathrm{N}^{\circ} 1496$.

Proponemos como alternativa de solución diseñar y ejecutar un plan para el trabajo de atención remota a los estudiantes, e institucionalizarlo mediante acuerdos del consejo universitario.

\section{DISEÑO METODOLOGICO}

La metodología que se utilizó para llevar a cabo la investigación, se sustentó en un tipo de indagación documental según Baena (2017) la investigación documental es una técnica utilizada para recopilar información de revistas, libros, informes, expedientes entre otros.

También se revisaron material bibliográfico que esta contenida en la tabla 1 en donde se detalla los documentos consultados por cantidad y tipo de documento.

Tabla 1. Material consultado en la investigación documental

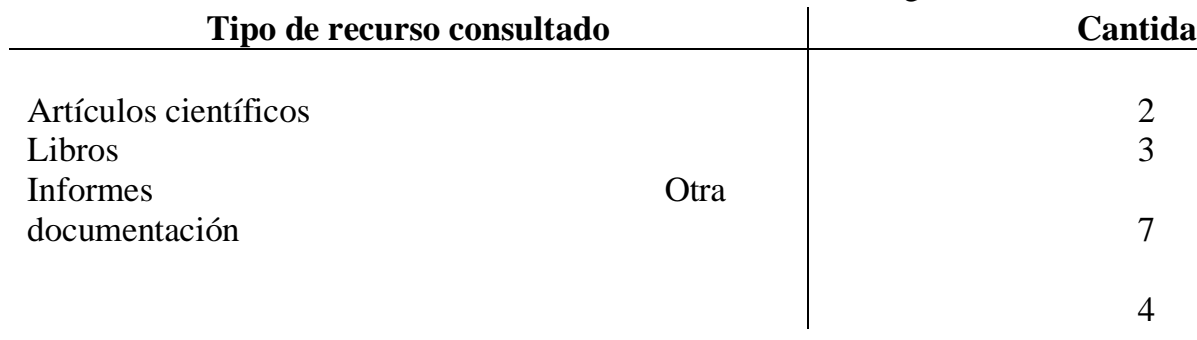

\section{RESULTADOS}

En la presente investigación se identificaron los siguientes hallazgos los cuales detallamos a continuación:

- Escasa implementación de políticas de Estado a largo plazo para la promoción de los recursos tecnológicos en las universidades.

- La Universidad ha sufrido un impacto económico negativo en sus ingresos económicos al ver limitado el uso de los servicios que brindan a terceros, producto de la paralización de las clases y el trabajo presencial en el marco de la emergencia sanitaria.

- Limitado acceso y conocimiento del uso de las nuevas TIC. fue una dificultad para el inicio y desarrollo trabajo virtual en el contexto de la crisis pandémica. 
- Escasa participación y promoción del cuidado, mediante las plataformas virtuales sobre temas relacionados al ahorro del agua, manejo de residuos sólidos, adecuado usos de energía, por parte de la comunidad universitaria

- Carencia de un plan institucional a largo plazo que aborde el tema del trabajo remoto por la emergencia sanitaria según el D.L. $\mathrm{N}^{\circ} 1496$.

\section{CONCLUSIONES Y RECOMENDACIONES}

Concluimos que la calidad educativa la Universidad en el contexto de la Globalización se manifiesta cuando:

La Universidad se convierte en un ente dinámico e innovador cuando implementa las políticas en su plan estratégico institucional para mejorar la calidad del servicio educativo, apostando por la implementación de currículos y sílabos por competencias, actualizados; así como la acreditación de las escuelas académicas, equipando ambientes y mejorando la infraestructura de los laboratorios, implementando los centros de investigación y fortaleciendo la actividad investigativa.

La Universidad debe fortalecer las competencias digitales docentes, lo que contribuirá en este tiempo de pandemia a la mejora en la atención a los estudiantes, formando profesionales competentes que puedan desempañarse en distintos escenarios laborales según las exigencias del mundo globalizado.

La universidad debe gestionar la firma de alianzas estratégicas con instituciones y universidades del país y con otras de índole internacional que permita el desarrollo de la ciencia y tecnologías a través de la formación de redes de comunidades científicas físicas o virtuales, así como también pasantías, intercambio académico de estudiantes, becas, entre otras.

Después de los hallazgos encontrados hacemos las siguientes recomendaciones

Implementar políticas para promover alianzas estratégicas con entidades universitarias del país y con otras de índole internacional que permita pasantías de estudiantes, becas, entre otras.

Adecuar los servicios presenciales que ofrece la Universidad a un entorno virtual en forma permanente a través de diferentes plataformas virtuales y modalidades de aprendizaje; así mismo incluir en los objetivos estratégicos e institucionalizar un plan para el logro de competencias relacionadas con el manejo de la tecnología en los docentes.

Promover campañas de propagación, concientización y capacitación en temas concernientes al manejo de desechos sólidos, a través de los diferentes medios y/o canales de difusión. 


\section{REFERENCIAS}

Alvarez, H. (2020). El Rol de la Tecnología en el nuevo contexto de COVID-19. USMP DIGITAL Revista de Facultad de CA y RRHH.(4). https://cutt.ly/hWNKLlk

Álvarez, C. (2020). Impacto del covid-19 sobre la Educación Superior. Consultado el 11 de setiembre de 2021 https://cutt.ly/8WNJz6F

Baena, G. (2017). Metodología de la investigación. Patria.

Comisión Económica para América Latina y el Caribe. (2020). El rol de los recursos naturales ante la pandemia por el COVID 19 en América Latina y el Caribe. CEPAL. https://cutt.ly/xWNGdjC

Diario Oficial El Peruano. (2020). Decreo supremo N002-2021 MINEDU. Ley De Presupuesto Del Sector del Público Para El Año Fiscal 2021. Consultado el 11 de setiembre de 2021 https://www.mef.gob.pe

Diario Oficial El Peruano. (2020). Decreto Legislativo N¹496. Disposiciones en Materia de Educación Superior universitaria en el marco del Estado de Emergencia Nacional. Consultado el 11 de setiembre de 2021 https://www.mef.gob.pe

Gibbons , M., Limoges, C., Nowothy, H., Schwartzman, S., Scott, P., \& Trow , M. (1998). La nueva producción del conocimiento (la dinámica de la ciencia y la investigación en las sociedades contemporáneas). Pomares.

Hernández, M. (2020). ¿El rostro del Perú? Identidad, diversidad y cambio. Agenda:Perú.

INEI. (2020). Comportamiento de la Economía Peruana en el 2020. Consultado el 11 de setiembre de 2021 https://www.inei.gob.pe/

Latinobarómetro, C. (2020). FICHA TÉCNICA 2020. LATINOBARÓMETRO. Consultado el 11 de setiembre de 2021 www.latinbaròmetro.org

Lombaerde, P., \& Lapade, P. (2012). Indicadores de la globalizaciòn. Cuadernos de economía. Consultado el 11 de setiembre de 2021 https://www.redalyc.org/articulo.oa?id=282124643001

MINEDU. (2019). Programa para la Evaluación Internacional de Estudiantes -PISA para el 2018. http://umc.minedu.gob.pe/pisa-2018

SUNEDU. (2020). Sunedu establece Condiciones Básicas de Calidad para la autorización de programas bajo las modalidades semipresencial y a distancia. www.sunedu.gob.pe UNAS. (2020). Resolución $N^{\circ} 206-2020-C U-R-U N A S$. https://portal.unas.edu.pe

Universidad Agraria de la Selva. (2019). Plan Estratégico Institucional Universidad Agraria de la Selva 2019-2023. Tingo Marìa: UNAS. https://portal.unas.edu.pe

Urquiola, M. (2020). Webinar El efecto disruptivo de la pandemia en la educación superior. (B. I. Desarrollo, Entrevistador) https://cutt.ly/RWNHDKn 